\title{
Involvement of Pattern Recognition Receptors in the Direct Influence of Bacterial Components and Standard Antiacne Compounds on Human Sebaceous Gland Cells
}

\author{
Zouboulis, Christos C ; Oeff, Marina K ; Hiroi, Naoki ; Makrantonaki, Evgenia ; Bornstein, Stefan R
}

\begin{abstract}
INTRODUCTION Pattern recognition receptors are involved in innate and adaptive immunity by detecting microbial components. Bacteria have been accused to play a role in inflammatory acne. We investigated the potential involvement of Toll-like receptor (TLR)2, TLR4, TLR6, and CD14 in the direct influence of bacterial components and standard antiacne compounds on human sebocytes. METHODS mRNA and protein expression of TLR2, TLR4, TLR6, and CD14 in SZ95 sebocytes was evaluated by real-time qRT-PCR and immunocytochemistry. The effects of lipopolysaccharides (LPS) and lipoteichoic acid on TLR2, TLR4, and CD14 expression and of cytokine/chemokine secretion by 13-cis-retinoic acid, all-trans-retinoic acid, retinol, and hydrocortisone at the mRNA and protein levels were assessed by realtime qRT-PCR and ELISA and verified by cocultivation with neutralizing antibodies. RESULTS The constitutive expression of TLR2, TLR4, and CD14 in SZ95 sebocytes was augmented by exposure to LPS. Hydrocortisone induced TLR2, but markedly reduced TLR4 expression. 13-cis-retinoic acid and all-transretinoic acid regulated IL-6 release. LPS enhanced and hydrocortisone reduced cytokine and chemokine release. Anti-TLR4 and anti-CD14 mAb blocked LPS-induced IL-8 and IL-6 release. CONCLUSIONS Microbial components use pattern recognition receptors to directly activate sebocytes to express a wide range of proinflammatory molecules and especially IL-8 and IL-6 in a TLR4- and CD14-specific manner. Retinoids, but mostly corticosteroids, also use this pathway to exhibit anti-inflammatory effects.
\end{abstract}

DOI: https://doi.org/10.1159/000513259

Posted at the Zurich Open Repository and Archive, University of Zurich

ZORA URL: https://doi.org/10.5167/uzh-208285

Journal Article

Published Version

Originally published at:

Zouboulis, Christos C; Oeff, Marina K; Hiroi, Naoki; Makrantonaki, Evgenia; Bornstein, Stefan R (2021). Involvement of Pattern Recognition Receptors in the Direct Influence of Bacterial Components and Standard Antiacne Compounds on Human Sebaceous Gland Cells. Skin Pharmacology and Physiology, 34(1):19-29.

DOI: https://doi.org/10.1159/000513259 


\title{
Involvement of Pattern Recognition Receptors in the Direct Influence of Bacterial Components and Standard Antiacne Compounds on Human Sebaceous Gland Cells
}

\author{
Christos C. Zouboulis ${ }^{a, b}$ Marina K. Oeff ${ }^{b, c}$ Naoki Hiroi ${ }^{c}$ \\ Evgenia Makrantonaki ${ }^{a}$,b Stefan R. Bornstein ${ }^{c}$
}

aDepartments of Dermatology, Venereology, Allergology and Immunology, Dessau Medical Center, Brandenburg Medical School Theodor Fontane and Faculty of Health Sciences Brandenburg, Dessau, Germany; ${ }^{b}$ Laboratory of Biogerontology, Dermato-Pharmacology and Dermato-Endocrinology, Departments of Dermatology, Venereology, Allergology and Immunology, Dessau Medical Center, Brandenburg Medical School Theodor Fontane and Faculty

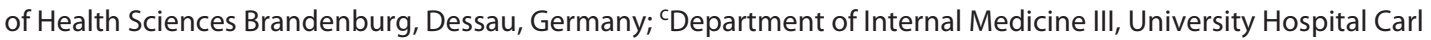
Gustav Carus, University of Dresden, Dresden, Germany

\section{Keywords}

Human sebaceous gland cells · Toll-like receptors .

Retinoids $\cdot$ Hydrocortisone $\cdot$ Innate immunity

\begin{abstract}
Introduction: Pattern recognition receptors are involved in innate and adaptive immunity by detecting microbial components. Bacteria have been accused to play a role in inflammatory acne. We investigated the potential involvement of Toll-like receptor (TLR)2, TLR4, TLR6, and CD14 in the direct influence of bacterial components and standard antiacne compounds on human sebocytes. Methods: mRNA and protein expression of TLR2, TLR4, TLR6, and CD14 in SZ95 sebocytes was evaluated by real-time qRT-PCR and immunocytochemistry. The effects of lipopolysaccharides (LPS) and lipoteichoic acid on TLR2, TLR4, and CD14 expression and of cytokine/chemokine secretion by 13-cis-retinoic acid, alltrans-retinoic acid, retinol, and hydrocortisone at the mRNA and protein levels were assessed by real-time qRT-PCR and ELISA and verified by cocultivation with neutralizing antibodies. Results: The constitutive expression of TLR2, TLR4, and CD14 in SZ95 sebocytes was augmented by exposure to
\end{abstract}

LPS. Hydrocortisone induced TLR2, but markedly reduced TLR4 expression. 13-cis-retinoic acid and all-trans-retinoic acid regulated IL-6 release. LPS enhanced and hydrocortisone reduced cytokine and chemokine release. Anti-TLR4 and anti-CD14 mAb blocked LPS-induced IL-8 and IL-6 release. Conclusions: Microbial components use pattern recognition receptors to directly activate sebocytes to express a wide range of proinflammatory molecules and especially IL-8 and IL-6 in a TLR4- and CD14-specific manner. Retinoids, but mostly corticosteroids, also use this pathway to exhibit anti-inflammatory effects.

(c) 2021 S. Karger AG, Basel

Christos C. Zouboulis and Marina K. Oeff joint co-authorship. The work presented in this manuscript has previously been reported in abstracts of the ESDR meeting 2002 (Oeff M.K., Seltmann H., Hakiy N., Bogdanoff B., Nastos A., Walters R., Bornstein S.R., Zouboulis C.C. Toll-like receptor 2 and 4-dependent regulation of inflammatory signaling in human sebocytes. J Invest Dermatol. 2002;119(3):736) and the EADV congress 2006 (Oeff M.K., Seltmann H., Hiroi N., Nastos A., Makrantonaki E., Bornstein S.R., Zouboulis C.C. Differential regulation of Toll-like receptor and CD14 pathways by retinoids and corticosteroids in human sebocytes. Dermatology. 2006;213:266). karger@karger.com

www.karger.com/spp

Karger

(n) (c) 2021 S. Karger AG, Base
Christos C. Zouboulis

Departments of Dermatology, Venereology, Allergollogy and Immunology Dessau Medical Center, Brandenburg Medical School Theodor Fontane and Faculty of Health Sciences Brandenburg, Auenweg 38, DE-06847 Dessau (Germany) christos.zouboulis@mhb-fontane.de 


\section{Introduction}

Human sebocytes are capable of bacterial recognition and may play an important role in initiating and perpetuating the activation of both innate and adaptive immune responses $[1,2]$. They participate in skin inflammation by recruiting and communicating with professional immune cells, an interaction, which leads to the generation and differentiation of Th17 cells [3]. Innate immune recognition of pathogens is mediated by germline-encoded receptors specific for conserved structural components of pathogenic microorganisms. These are referred to as pattern recognition receptors (PRR) [4]. Genome-wide analysis of PRR-activated human sebocytes presented a complex immune competence of activated sebaceous glands [5]. Human acne-involved sebaceous glands were shown to express common molecules with other inflammatory diseases, such as psoriasis and lichen ruber [6].

Toll-like receptors (TLR) act as PRR. TLR are transmembrane proteins with an extracellular domain consisting of leucine-rich repeats and cytoplasmic domain similar to the interleukin (IL)-1 receptor and have emerged as key components in the innate immune system [7]. TLR recognize conserved molecular structures, known as pathogen-associated molecular patterns, which are relatively invariant within a given class of microorganisms such as mannans in the yeast cell wall, mycobacterial cellwall component lipoarabinomannan, lipopolysaccharides (LPS) in Gram-negative bacteria, and lipoteichoic acid (LTA) in Gram-positive bacteria. TLR play a crucial role in the induction of antimicrobial responses in various cells activating multiple steps in those inflammatory reactions that help to eliminate the invading bacteria and coordinate systemic defense [8].

The possible role of TLR in acne pathogenesis has been investigated by targeting Propionibacterium acnes ( $P$. acnes) as the most obvious source of the pathogen-associated molecular pattern recognized by TLR2 $[2,9,10]$. TLR2 and TLR4 as well as CD14 are expressed in human monocytes. TLR2 is upregulated on perilesional macrophages of acne lesions, and $P$. acnes can activate TLR 2 and trigger IL-8 release from peripheral blood mononuclear cells (PBMC) [11].

IL-8 is chemotactic for neutrophils, and early acne lesions are characterized by a paucity of neutrophils [12, 13]. They are most likely to be involved later in the generation of pustules. Several putative physiological ligands of TLR, such as - most intriguingly - fatty acids, are likely to be involved in acne pathogenesis $[14,15]$.
TLR, such as TLR2 and TLR4, have also been identified activated on human epidermal keratinocytes $[16,17]$ and sebocytes in vitro $[5,18,19]$. Moreover, TLR2 is expressed in basal and infundibular keratinocytes and in sebaceous glands in vivo and its activation provoked the release of IL-1 a from primary human keratinocytes in vitro. The exposure of microdissected human sebaceous glands to the TLR ligands LPS, peptidoglycan, and LTA resulted in a pattern of IL-1 $\alpha$-like cornification after 7 days of exposure [20]. Clinical isolates of $P$. acnes significantly induced IL-8 mRNA expression in human keratinocytes in vitro [21]. P. acnes-induced increase in IL-8 gene expression could be inhibited by anti-TLR2 and anti-TLR4 neutralizing antibodies.

A major target of acne therapy is the reduction of inflammation. Retinoids and hydrocortisone (HC) have shown anti-inflammatory activity in the treatment of severe acne [22-24], whereas dexamethasone increased TLR2 mRNA expression in sebocytes by inhibiting $11 \beta$-hydroxysteroid dehydrogenase type 1 and activating mitogen-activated protein kinase phosphatase-1 [18]. Treatment of primary human monocytes with all-trans retinoic acid (atRA) led to downregulation of TLR2 and the TLR4 coreceptor CD14 [25]. The ability of a TLR2/1 ligand to trigger monocyte cytokine release was inhibited by pretreatment and co-treatment with atRA, which also downregulated monocyte cytokine induction by P. acnes. At last, systemic isotretinoin (13-cis-retinoic acid [13cRA]) therapy normalized the exaggerated TLR2-mediated innate immune response in acne patients [26]. The goal of the present study was to investigate or confirm the potential involvement of Toll-like receptor (TLR)2, TLR4, TLR6, and CD14 in the direct influence of bacterial components and standard antiacne compounds on human sebocytes in vitro.

\section{Materials and Methods}

\section{Cell Culture}

Immortalized human facial SZ95 sebocytes [27], which have been shown to conserve the major characteristics of normal sebocytes [28], were maintained in Sebomed ${ }^{\circledR}$ Basal Medium (Biochrom, Berlin, Germany) supplemented with $10 \%$ heat-inactivated fetal calf serum (Biochrom), $5 \mathrm{ng} / \mathrm{mL}$ human epidermal growth factor (Sigma, Deisenhofen, Germany), $50 \mu \mathrm{g} / \mathrm{mL}$ gentamicin (Gibco/BRL, Berlin, Germany), and $1 \mathrm{mM} \mathrm{Ca}^{2+}$ in a humidified atmosphere of $5 \% \mathrm{CO}_{2}$ at $37^{\circ} \mathrm{C}$. Cell culture medium was changed every 2 days. SZ95 sebocytes were allowed to grow to $70 \%$ confluence. The human acute myeloid leukemia cell line HL-60 [29] was cultured under the same conditions with SZ95 sebocytes. PBMC were prepared from peripheral blood from healthy individuals as 
previously described [30]. HL-60 cells and PBMC served as positive controls. Keratinocytes from neonatal foreskin cultured in serum-free keratinocyte medium (Gibco/BRL) supplemented with $5 \mathrm{ng} / \mathrm{mL}$ epidermal growth factor, $50 \mu \mathrm{g} / \mathrm{mL}$ bovine pituitary extract, and $50 \mu \mathrm{g} / \mathrm{mL}$ gentamicin (all from Gibco/BRL) in a humidified atmosphere of $5 \% \mathrm{CO}_{2}$ at $37^{\circ} \mathrm{C}$ served as additional control. Since the cells used in this study either originate from immortalized human cell lines or from previously established normal human cell lines and did not derive from tissue specimens of human subjects obtained for the presented studies no written concent or ethics approval was required.

\section{Reagents}

For in vitro stimulation of SZ95 sebocytes, the following substances were used: 13cRA, atRA, retinol (ROL), HC, LPS from Escherichia coli 026:B6, and LTA from Bacillus subtilis. Phorbol 12-myristate 13-acetate (PMA) served as positive control (all from Sigma). Mouse monoclonal antibodies (mAb) were used for immunocytochemistry and neutralization of microbial componentinduced chemokine and cytokine production: anti-human CD14 (OriGene, Rockville, MD, USA), anti-human TLR2 TL2.1 (Thermo Fisher, Waltham, MA, USA), anti-human TLR4 HTA125 (Novus, Centennial, CO, USA), and IgG2a isotype control (Thermo Fischer). All secondary antibodies used and the alkaline phosphatase anti-alkaline phosphatase (APAAP) complex were from Dako (Hamburg, Germany). ELISA kits were from R\&D Systems (Wiesbaden, Germany).

\section{Cell Challenge}

SZ95 sebocytes grown to about $70 \%$ confluence were maintained further under serum-free conditions and were either left untreated or challenged for $24 \mathrm{~h}$ with $10^{-6} \mathrm{M} 13 \mathrm{cRA}, 10^{-6} \mathrm{M}$ atRA, $10^{-6} \mathrm{M}$ ROL, $10^{-6} \mathrm{M}$ HC, $100 \mathrm{ng} / \mathrm{mL}$ LPS, $100 \mathrm{ng} / \mathrm{mL}$ LTA, and 100 $\mathrm{ng} / \mathrm{mL}$ PMA. Following incubation, the supernatants were collected and the cells were harvested for detection of mRNA and protein levels.

\section{RT-PCR Analysis}

Total RNA was extracted from SZ95 sebocytes, keratinocytes, PBMC, and HL-60 by standard methods (RNeasy Midi Kit; Qiagen, Hilden, Germany). The total RNA was then treated with RNase-Free DNase Set (Qiagen). An aliquot of total RNA (1 $\mu \mathrm{g})$ was reverse-transcribed into single-stranded cDNA with oligo(dT)15 (1 $\mu \mathrm{g})$ and Moloney murine leukemia virus reverse transcriptase in a final volume of $33 \mu \mathrm{L}$ as indicated in the manufacturer's instructions (First-strand cDNA Synthesis Kit; GE Healthcare, Freiburg, Germany). Reverse transcription mixture (2 $\mu \mathrm{L}$ ) was used for the PCR reaction. PCR was carried out in a total volume of $50 \mu \mathrm{L}$ with a primer pair at $0.5 \mu \mathrm{M}$ in $50 \mathrm{mM} \mathrm{KCl}, 10 \mathrm{mM}$ Tris- $\mathrm{HCl}$ (pH 8.3), $1.5 \mathrm{mM} \mathrm{MgCl}_{2}, 200 \mu \mathrm{M}$ each of dATP, dCTP, dTTP, and dGTP, and 1.25 units Ampli Tag DNA polymerase (Applied Biosystems, Weiterstadt, Germany) in an Eppendorf Mastercycler gradient-automated thermal cycler. The amplification protocol contained 1 cycle of initial denaturation at $94^{\circ} \mathrm{C}$ for $5 \mathrm{~min}$ followed by varying number of cycles of denaturation at $94^{\circ} \mathrm{C}$ for $1 \mathrm{~min}$, annealing at the individual annealing temperature $\left(T_{\mathrm{a}}\right)$ for $1 \mathrm{~min}$, extension at $72^{\circ} \mathrm{C}$ for $2 \mathrm{~min}$, and 1 cycle of terminal extension at $72^{\circ} \mathrm{C}$ for $10 \mathrm{~min}$. PCR products were separated electrophoretically on 1.5-2\% Tris-acetate EDTA-agarose gels, stained with ethidium bromide, and photographed under UV light. Table 1

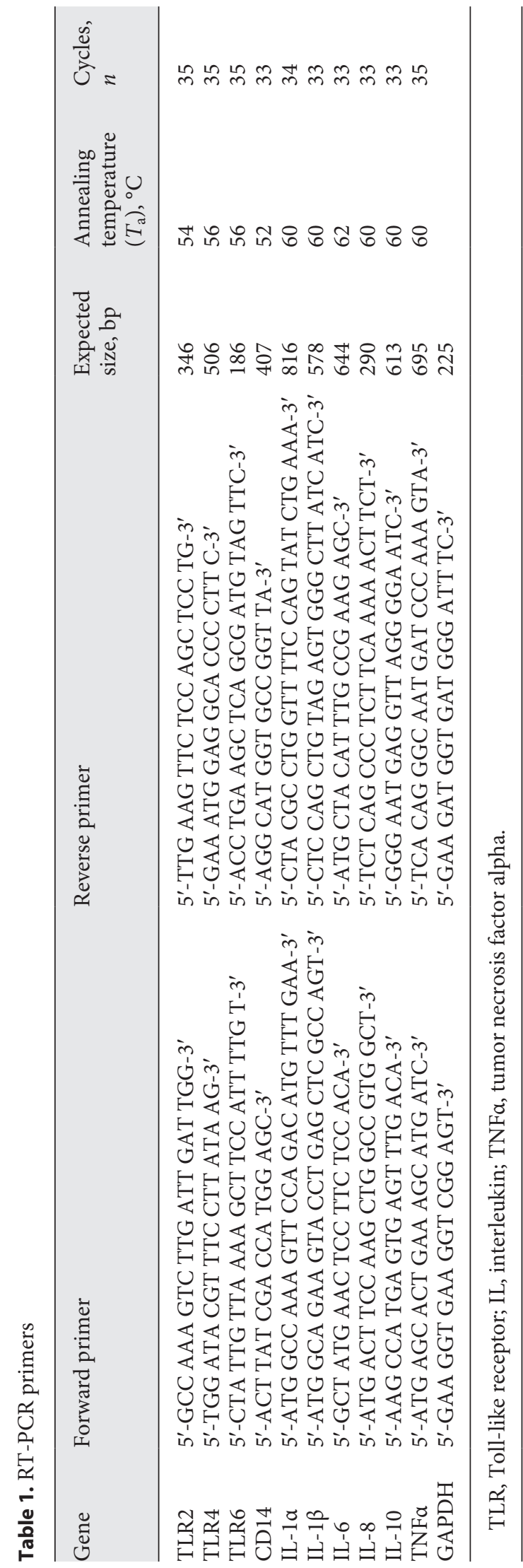

Skin Pharmacol Physiol 2021;34:19-29

DOI: $10.1159 / 000513259$ 

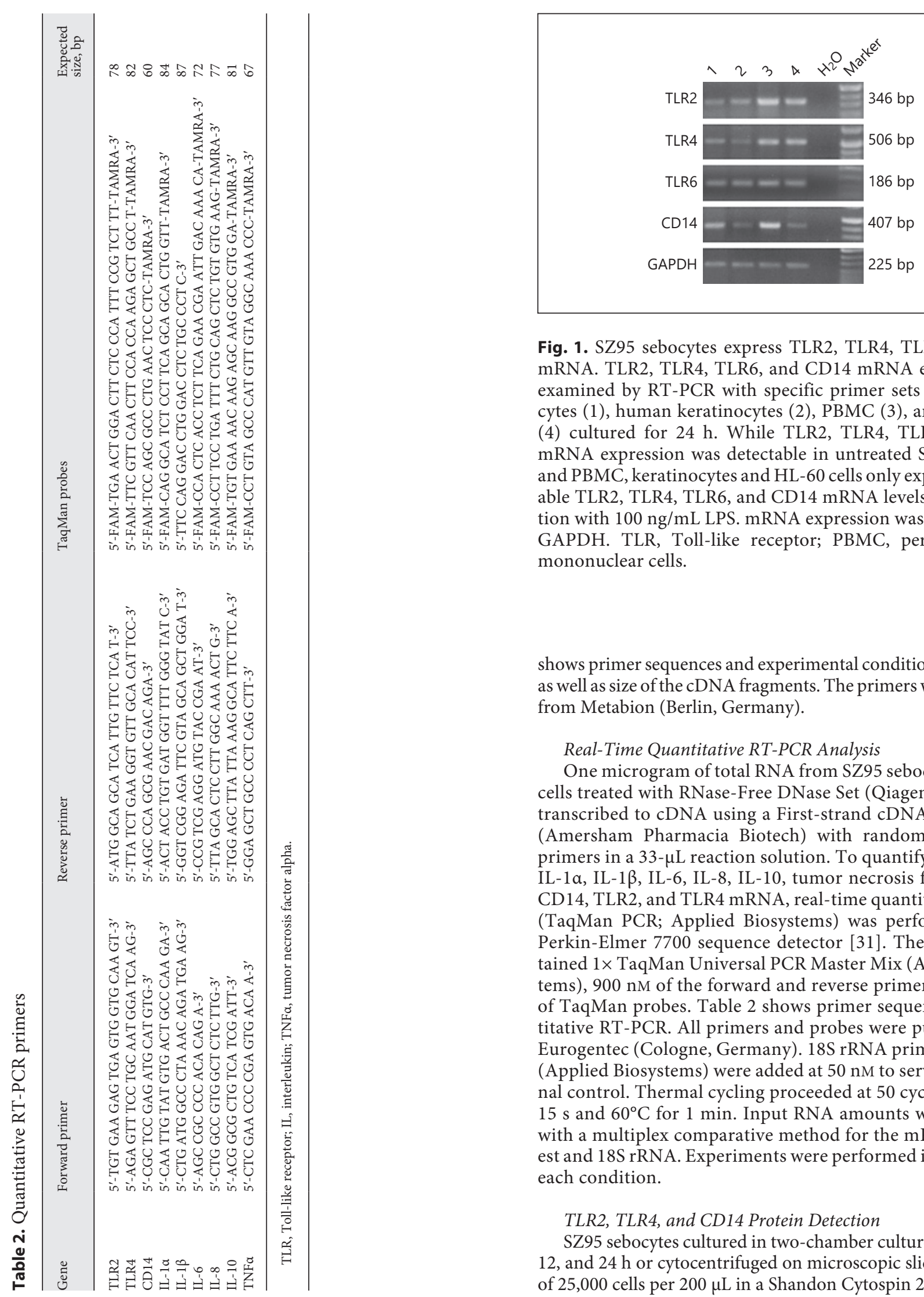

Fig. 1. SZ95 sebocytes express TLR2, TLR4, TLR6, and CD14 mRNA. TLR2, TLR4, TLR6, and CD14 mRNA expression was examined by RT-PCR with specific primer sets in SZ95 sebocytes (1), human keratinocytes (2), PBMC (3), and HL-60 cells (4) cultured for $24 \mathrm{~h}$. While TLR2, TLR4, TLR6, and CD14 mRNA expression was detectable in untreated SZ95 sebocytes and PBMC, keratinocytes and HL-60 cells only expressed detectable TLR2, TLR4, TLR6, and CD14 mRNA levels after stimulation with $100 \mathrm{ng} / \mathrm{mL}$ LPS. mRNA expression was normalized to GAPDH. TLR, Toll-like receptor; PBMC, peripheral blood mononuclear cells.

shows primer sequences and experimental conditions for RT-PCR as well as size of the cDNA fragments. The primers were purchased from Metabion (Berlin, Germany).

\section{Real-Time Quantitative RT-PCR Analysis}

One microgram of total RNA from SZ95 sebocytes or HL-60 cells treated with RNase-Free DNase Set (Qiagen) was reversetranscribed to cDNA using a First-strand cDNA Synthesis Kit (Amersham Pharmacia Biotech) with random hexamers as primers in a $33-\mu \mathrm{L}$ reaction solution. To quantify expression of IL-1 $\alpha$, IL-1 $\beta$, IL-6, IL-8, IL-10, tumor necrosis factor (TNF $\alpha$ ), CD14, TLR2, and TLR4 mRNA, real-time quantitative RT-PCR (TaqMan PCR; Applied Biosystems) was performed using a Perkin-Elmer 7700 sequence detector [31]. The reaction contained $1 \times$ TaqMan Universal PCR Master Mix (Applied Biosystems), $900 \mathrm{nM}$ of the forward and reverse primers, and $200 \mathrm{nM}$ of TaqMan probes. Table 2 shows primer sequences for quantitative RT-PCR. All primers and probes were purchased from Eurogentec (Cologne, Germany). 18S rRNA primers and probe (Applied Biosystems) were added at $50 \mathrm{nM}$ to serve as the internal control. Thermal cycling proceeded at 50 cycles of $95^{\circ} \mathrm{C}$ for $15 \mathrm{~s}$ and $60^{\circ} \mathrm{C}$ for $1 \mathrm{~min}$. Input RNA amounts were calculated with a multiplex comparative method for the mRNAs of interest and 18S rRNA. Experiments were performed in triplicate for each condition.

\section{TLR2, TLR4, and CD14 Protein Detection}

SZ95 sebocytes cultured in two-chamber culture slides for 1, 6, 12 , and $24 \mathrm{~h}$ or cytocentrifuged on microscopic slides at a density of 25,000 cells per $200 \mu \mathrm{L}$ in a Shandon Cytospin 2 cytocentrifuge 

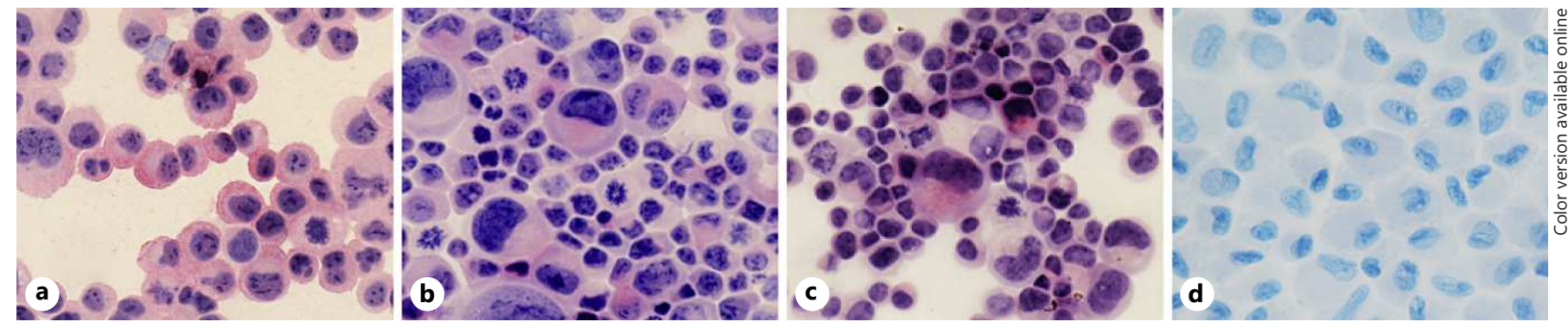

Fig. 2. Human SZ95 sebocytes express TLR2, TLR4, and CD14 protein. Protein expression was examined by immunocytochemistry with mouse anti-human TLR2 (a), TLR4 (b), and CD14 (c) antibodies. d Unlabeled control. TLR 2 could be mainly attributed to the cell membrane, while TLR4 and CD14 were mainly distributed diffusely in the cytoplasm of SZ95 sebocytes. TLR, Toll-like receptor.

were subjected to immunocytochemistry. The preparations were fixed in methanol at $-20^{\circ} \mathrm{C}$ for $5 \mathrm{~min}$ and incubated with antiCD14 (diluted 1:50), anti-TLR2 (diluted 1:20), or anti-TLR4 (diluted 1:20) antibodies for $30 \mathrm{~min}$. The secondary antibody used was APAAP complex-conjugated rabbit anti-mouse (diluted 1:20), and the reaction was performed according to the instructions for the APAAP labeling method (all incubation steps at room temperature for $30 \mathrm{~min}$ ). The preparations were developed with new fuchsine counterstained with hematoxylin and mounted. Triplicate slides were stained for each data point.

\section{Determination of $I L-1 \alpha, I L-1 \beta, I L-6, I L-8, I L-10$, and TNF $\alpha$} Release

Chemokines and cytokines were measured in the supernatants of challenged SZ95 sebocytes using Quantikine ELISA kits (R\&D System, Wiesbaden, Germany) according to the manufacturer's protocols. IL and TNFa release was measured in triplicate wells for all individual treatments. The results are presented as the relative fold increase compared to against the results from the untreated controls at the same time, whereas the ratio 1 represents the IL release of the controls.

Neutralization of Microbial Component-Induced Chemokine and Cytokine Production by Anti-TLR2, Anti-TLR4, and Anti-CD14 Antibodies

Cultured cells were preincubated for $1 \mathrm{~h}$ at $37^{\circ} \mathrm{C}$ with $10 \mu \mathrm{g}$ per $\mathrm{mL}$ anti-TLR2, anti-TLR4, or anti-CD14 antibodies followed by the addition of $100 \mathrm{ng} / \mathrm{mL}$ LPS, LTA, or PMA. To determine the specificity of these antibody studies, control cultures were preincubated with a mouse IgG2a isotype control antibody followed by exposure to $100 \mathrm{ng} / \mathrm{mL}$ LPS, LTA, or PMA. After $24 \mathrm{~h}$, the culture supernatants were harvested and assessed by Quantikine ELISA kits according to the manufacturer's protocols. Details of proliferation, differentiation, or viability of SZ95 sebocytes are given in online suppl. data; for all online suppl. material, see www. karger.com/doi/10.1159/000513259.

\section{Statistical Analysis}

All data are presented as the mean \pm SEM for at least 3 experiments. Statistical significance was calculated by the Yates methodcorrected two-sample independent-group Student's $t$ test. Mean differences were considered significant at values of $p<0.05$.

Toll-Like Receptors in Human Sebocytes

\section{Results}

SZ95 Sebocytes Express TLR2, TLR4, TLR6, and CD14 at the MRNA Level

TLR2, TLR4, TLR6, and CD14 mRNA were detected in SZ95 sebocytes by RT-PCR and their levels, especially those of TLR2 and CD14, increased with the duration of SZ95 sebocyte culture (Fig. 1). PBMC, LPS-stimulated HL-60 cells, and epidermal keratinocytes served as positive controls and sterilized water as negative control. We detected TLR2, TLR4, TLR6, and CD14 mRNA expression in SZ95 sebocytes and all positive controls.

\section{Expression of TLR2, TLR4, and CD14 at the}

Protein Level in SZ95 Sebocytes and Their Cellular

Localization

Labeling of SZ95 sebocytes with anti-TLR2, antiTLR4, and anti-CD14 mAb revealed a positive staining for all molecules tested (Fig. 2). TLR2 could be mainly attributed to the cell membrane, while TLR4 and CD14 were mainly distributed diffusely in the cytoplasm of SZ95 sebocytes. These results have been corroborated by Western blot detection of abundant TLR 2 and TLR4 protein levels in SZ95 sebocytes, shown in another own work submitted for publication.

\section{Effect of LPS and LTA on TLR2, TLR4, and CD14} $m R N A$ Levels in SZ95 Sebocytes

As expected, the proinflammatory/cytotoxic agent PMA used as positive control markedly upregulated TLR2 $(2.55 \pm 0.77 \mathrm{x})$ and TLR4 $(2.63 \pm 0.58 \mathrm{x})$ mRNA levels in SZ95 sebocytes, being the only compound tested to induce an increase in mRNA levels of over 2.5-fold in all experiments performed (Fig. 3). Challenging SZ95 sebocytes with LPS slightly increased mRNA levels of TLR2 


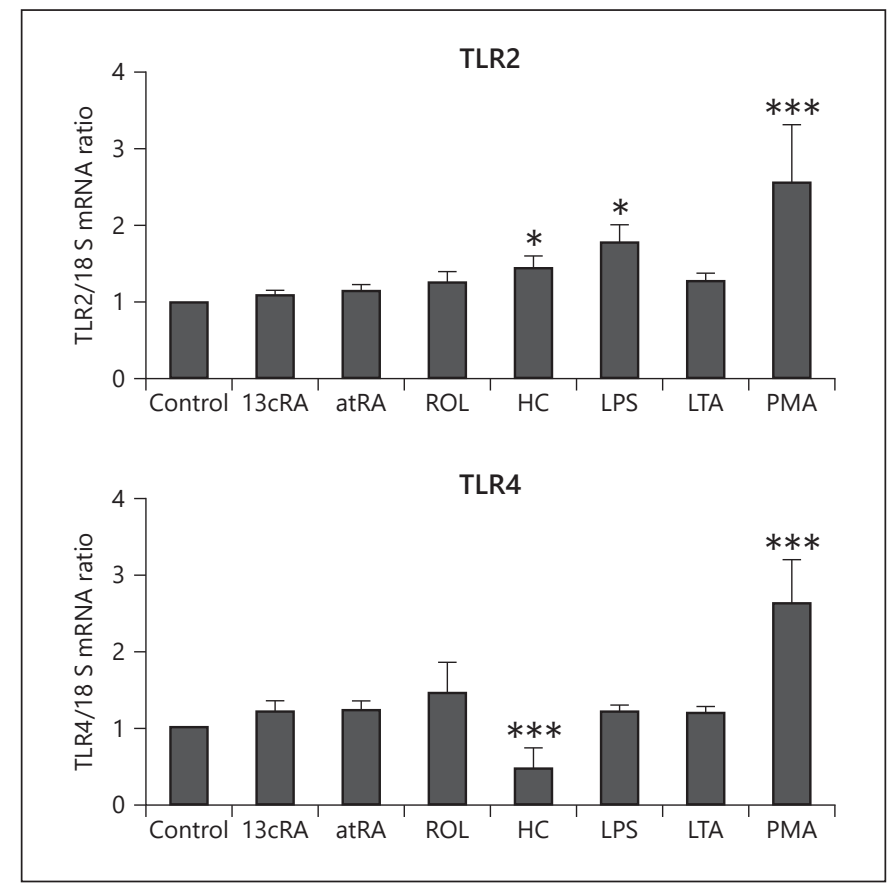

Fig. 3. TLR2 and TLR4 mRNA levels in SZ95 sebocytes are regulated by microbial components and anti-inflammatory agents. TLR2 and TLR4 mRNA levels in SZ95 sebocytes were assessed by real-time quantitative RT-PCR $24 \mathrm{~h}$ after treatment with $10^{-6} \mathrm{M}$ $13 \mathrm{cRA}, 10^{-6} \mathrm{M}$ atRA, $10^{-6} \mathrm{M}$ ROL, $10^{-6} \mathrm{M} \mathrm{HC}, 100 \mathrm{ng} / \mathrm{mL}$ LPS, $100 \mathrm{ng} / \mathrm{mL}$ LTA, and $100 \mathrm{ng} / \mathrm{mL}$ PMA. TLR2 and TLR4 mRNA levels were normalized with $18 \mathrm{~S}$ rRNA levels and are presented as the $\mathrm{x}$-fold increase over the untreated controls at $24 \mathrm{~h}$. LPS upregulated TLR2 mRNA levels. HC slightly upregulated TLR2 mRNA levels but markedly reduced TLR4 mRNA levels. PMA markedly upregulated TLR2 and TLR4 mRNA levels in SZ95 sebocytes. The chart shows the mean \pm SEM of 10 independent experiments. Statistical analysis was performed by Student's $t$ test using a two-tailed hypothesis. ${ }^{*} p<0.05$; ${ }^{* * *} p<0.01$. TLR, Toll-like receptor; 13cRA, 13-cis-retinoic acid; atRA, all-trans retinoic acid; ROL, retinol; HC, hydrocortisone; LPS, lipopolysaccharides; LTA, lipoteichoic acid; PMA, phorbol 12-myristate 13-acetate.

$(1.77 \pm 0.24 \mathrm{x})$, while LTA did not affect TLR2 and TLR4 mRNA levels. CD14 mRNA levels were affected by neither LPS nor LTA, indicating CD14 is present in human sebocytes as a co-player for the induction of inflammatory processes by TLR.

Effect of Retinoids and HC on TLR2, TLR4, and CD14 mRNA Levels in SZ95 Sebocytes

TLR2, TLR4, and CD14 mRNA levels in SZ95 sebocytes were assessed after treatment with the retinoids $13 \mathrm{cRA}$, atRA, and ROL as well as with $\mathrm{HC}$, compounds known to modify the inflammatory response in vivo and in vitro (Fig. 3). All retinoids tested did not affect TLR2 and TLR4 mRNA levels. HC slightly enhanced TLR2 mRNA levels $(1.44 \pm 0.17 \mathrm{x})$ and reduced TLR4 mRNA levels $(0.46 \pm 0.20 \mathrm{x})$. We could reproduce this HC effect with additional experiments using dexamethasone (data not shown).

\section{Effect of Microbial Components on Cytokine and}

Chemokine Synthesis in SZ95 Sebocytes

To detect the effects of LPS and LTA on cytokine/chemokine synthesis in SZ95 sebocytes, we measured the production of selected proinflammatory chemokines and cytokines by real-time quantitative RT-PCR and ELISA. The positive control substance PMA increased IL-1 $\alpha$, IL$1 \beta$, IL-6, and IL- 8 mRNA levels and protein secretion (IL$1 \alpha[4.44 \pm 0.76 \mathrm{x}], \mathrm{IL}-1 \beta[4.94 \pm 0.44 \mathrm{x}], \mathrm{IL}-6$ [4.34 $\pm 0.68 \mathrm{x}]$, and especially IL-8 secretion levels [32.25 $\pm 15.90 \mathrm{x}]$ ) from SZ95 sebocytes (Fig. 4). In contrast to LTA treatment, which did not affect cytokine and chemokine protein levels, LPS enhanced cytokine and chemokine release (IL-1a $[1.65 \pm 0.15 x]$, IL-1 $\beta[2.08 \pm 0.20 x]$, IL-6 $[2.97 \pm 0.40 x]$, and IL-8 $[4.83 \pm 1.11 \mathrm{x}])$ from SZ95 sebocytes. IL-10 and TNF $\alpha$ mRNA expression and release were only markedly induced by PMA, but not by LPS or LTA.

\section{Effect of Retinoids and HC on Cytokine and}

Chemokine Synthesis in SZ95 Sebocytes

To detect the effects of retinoids and HC on cytokine/ chemokine synthesis in SZ95 sebocytes, we also measured their effect on the production of selected proinflammatory chemokines and cytokines by real-time quantitative RT-PCR and ELISA. As expected from the TLR pattern regulation reported above, retinoids did not affect mRNA levels of IL-1 $\alpha$, IL- $1 \beta$, IL- 6 , and IL- 8 and only induced minor effects on cytokine/chemokine release (Fig. 4). $13 \mathrm{cRA}$ slightly reduced IL- $1 \alpha(0.83 \pm 0.03 \mathrm{x})$ and IL-6 $(0.42 \pm 0.10 \mathrm{x})$ secretion and atRA IL-6 $(0.50 \pm 0.10 \mathrm{x})$ and ROL IL-1 $\alpha(0.71 \pm 0.10 \mathrm{x})$ secretion from SZ95 sebocytes. In contrast, $\mathrm{HC}$ reduced cytokine and chemokine release (IL- $1 \alpha[0.45 \pm 0.08 x]$, IL- $1 \beta[0.59 \pm 0.05 x]$, IL-6 [0.28 \pm $0.04 \mathrm{x}]$, and IL-8 [0.26 $\pm 0.06 \mathrm{x}]$ ) from SZ95 sebocytes. IL10 and TNFa release was affected neither by retinoids nor by HC.

LPS Induces SZ95 Sebocyte IL-8 and IL-6 Release by a

TLR4- and CD14-Dependent Mechanism

To determine whether IL-8 and IL-6 synthesis in SZ95 sebocytes is regulated by TLRs and/or CD14, we investigated the effect of anti-TLR2, anti-TLR4, and anti-CD14 antibodies on LPS- and LTA-induced IL- 8 and IL- 6 re- 


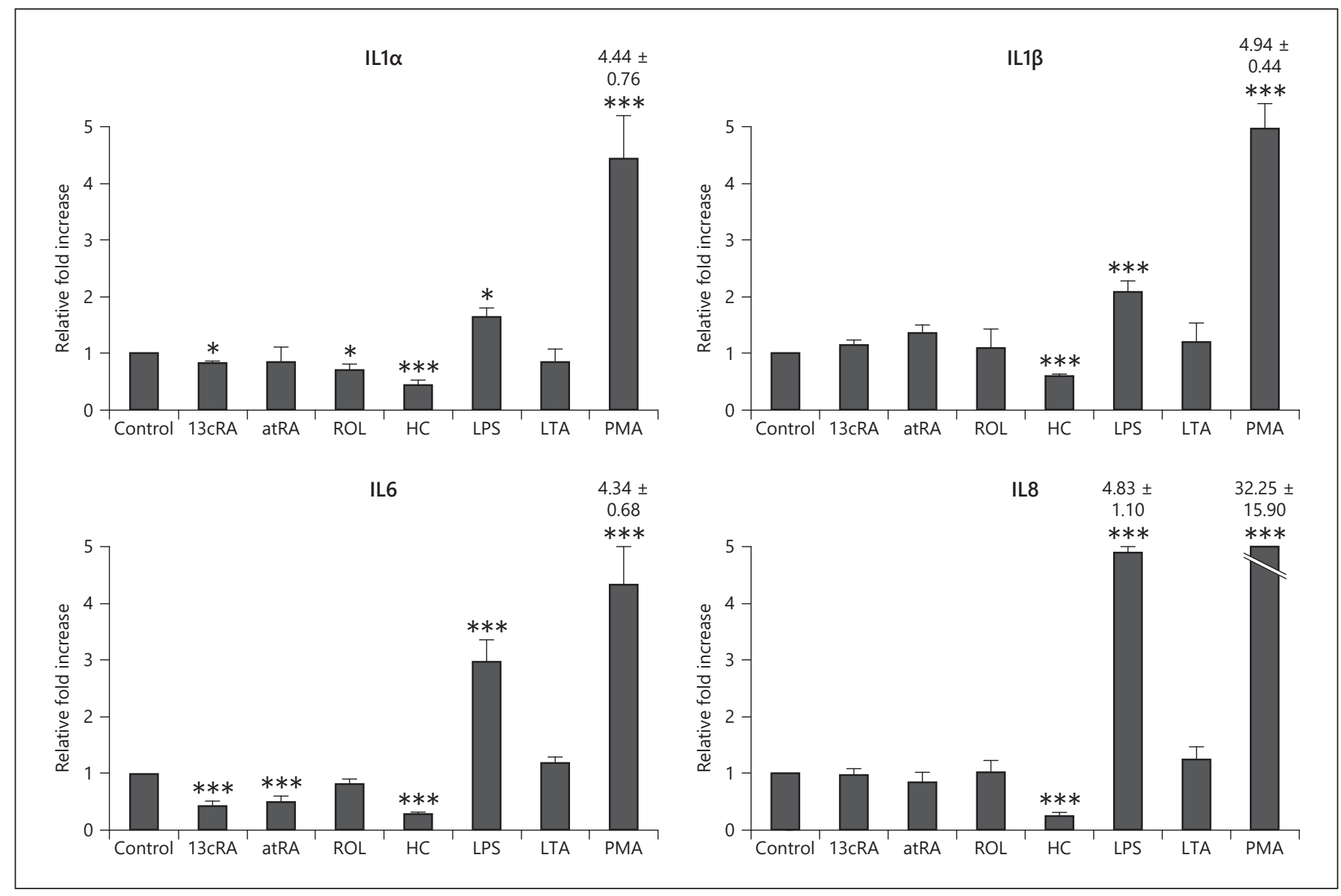

Fig. 4. Cytokine and chemokine production in SZ95 sebocytes is regulated by microbial components and anti-inflammatory agents. The effect of $10^{-6} \mathrm{M} 13 \mathrm{cRA}, 10^{-6} \mathrm{M}$ atRA, $10^{-6} \mathrm{M}$ ROL, $10^{-6} \mathrm{M} \mathrm{HC}$, $100 \mathrm{ng} / \mathrm{mL}$ LPS, $100 \mathrm{ng} / \mathrm{mL}$ LTA, and $100 \mathrm{ng} / \mathrm{mL}$ PMA on IL- $1 \alpha$, IL-1 $\beta$, IL-6, and IL- 8 release from SZ95 sebocytes was measured by ELISA in 24-h cell culture supernatants. In contrast to LTA, which was found inactive, LPS enhanced and HC reduced the release of all cytokines and chemokines tested. 13cRA reduced IL-1 $\alpha$ and IL-6 secretion and atRA IL-6 and ROL IL-1 $\alpha$ secretion from SZ95

lease. No effect was detected on LPS- and LTA-induced SZ95 sebocyte IL- 8 and IL-6 release after incubation with the IgG2a isotype antibody used as negative control. As expected, PMA-induced IL- 8 and IL- 6 release used as the positive control was not affected by incubation of SZ95 sebocytes with anti-TLR2, anti-TLR4, and anti-CD14 antibodies (Fig. 5, 6). IL-8 release induced by LPS was reduced by anti-TLR $4 \mathrm{mAb}(4.28 \pm 0.89$ to $1.80 \pm 0.35 \mathrm{x})$, as expected and given the lack of IL- 8 stimulation by LTA basal IL- 8 release was slightly reduced by anti-TLR $2 \mathrm{mAb}$ $(1.30 \pm 0.19$ to $0.74 \pm 0.09 x)$ (Fig. 5). The anti-CD14 mAb caused a reduction in IL-8 release by LPS ( $4.78 \pm 1.05$ to

sebocytes. PMA markedly upregulated the secretion of all cytokines tested. Data are presented as the $\mathrm{x}$-fold increase over the untreated control at $24 \mathrm{~h}$. The chart shows the mean \pm SEM of 7 independent experiments. Statistical analysis was performed by Student's $t$ test using a two-tailed hypothesis. ${ }^{*} p<0.05 ;{ }^{* * *} p<0.01$. 13cRA, 13-cis-retinoic acid; atRA, all-trans retinoic acid; ROL, retinol; HC, hydrocortisone; LPS, lipopolysaccharides; LTA, lipoteichoic acid; PMA, phorbol 12-myristate 13-acetate.

$3.14 \pm 0.40 \mathrm{x})$, as also expected since CD14 is a co-receptor for TLR4. Moreover, anti-TLR4 and anti-CD14 mAb inhibited IL-6 release induced by LPS (anti-TLR4: $2.74 \pm$ 0.34 to $1.72 \pm 0.26 \mathrm{x}$; anti-CD14: $3.62 \pm 0.52$ to $1.42 \pm$ $0.26 \mathrm{x})$. Given the lack of IL- 6 stimulation by LTA, antiTLR4 and anti-CD14 mAb seemed to also slightly reduce the basal IL-6 release (anti-TLR4: $1.21 \pm 0.11$ to $0.51 \pm$ $0.17 \mathrm{x}$; anti-CD14: $1.26 \pm 0.21$ to $0.50 \pm 0.23 \mathrm{x}$ ), respectively (Fig. 6). On the other hand, incubation with the antiTLR2 antibody inhibited neither IL-8 nor IL-6 release induced by LPS in SZ95 sebocytes (Fig. 5, 6). 


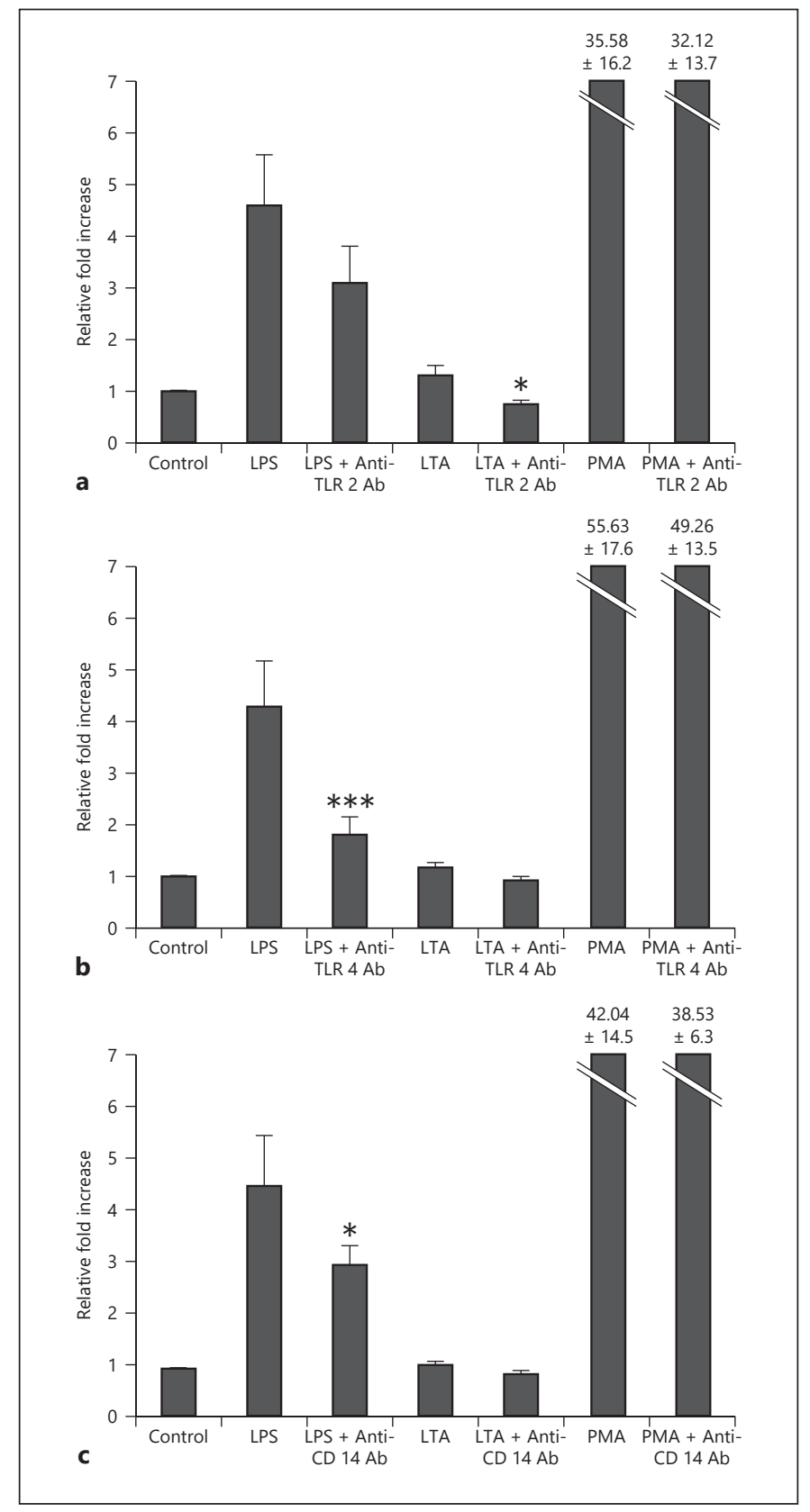

Fig. 5. Microbial components induce SZ95 sebocytes to release IL- 8 by a TLR4- and CD14-dependent mechanism. LPS and LTA induction of SZ95 sebocyte IL-8 secretion was examined by ELISA. SZ95 sebocytes were pretreated with $10 \mu \mathrm{g} / \mathrm{mL}$ anti-TLR2 $\mathrm{mAb}(\mathbf{a})$, anti-TLR4 mAb (b), anti-CD14 mAb, (c) or mouse IgG2a isotype control antibody for $1 \mathrm{~h}$ and then challenged with $100 \mathrm{ng} / \mathrm{mL}$ LPS, LTA, or PMA for $24 \mathrm{~h}$. Anti-TLR4 $\mathrm{mAb}$ and anti-CD14 mAb blocked LPS-induced but not LTA-induced IL-8 release. Anti-TLR2 mAb slightly reduced basal IL-8 release. Control IL-8 levels (under IgG2a isotype control antibody treatment): $339 \pm 28 \mathrm{pg} / \mathrm{mL}$. The chart shows the mean \pm SEM of 7 independent experiments. Statistical analysis was performed by Student's $t$ test using a two-tailed hypothesis. ${ }^{*} p<0.05$; ${ }^{* * *} p<0.01$. TLR, Toll-like receptor; LPS, lipopolysaccharides; LTA, lipoteichoic acid; PMA, phorbol 12-myristate 13-acetate; $\mathrm{mAb}$, mouse monoclonal antibodies.

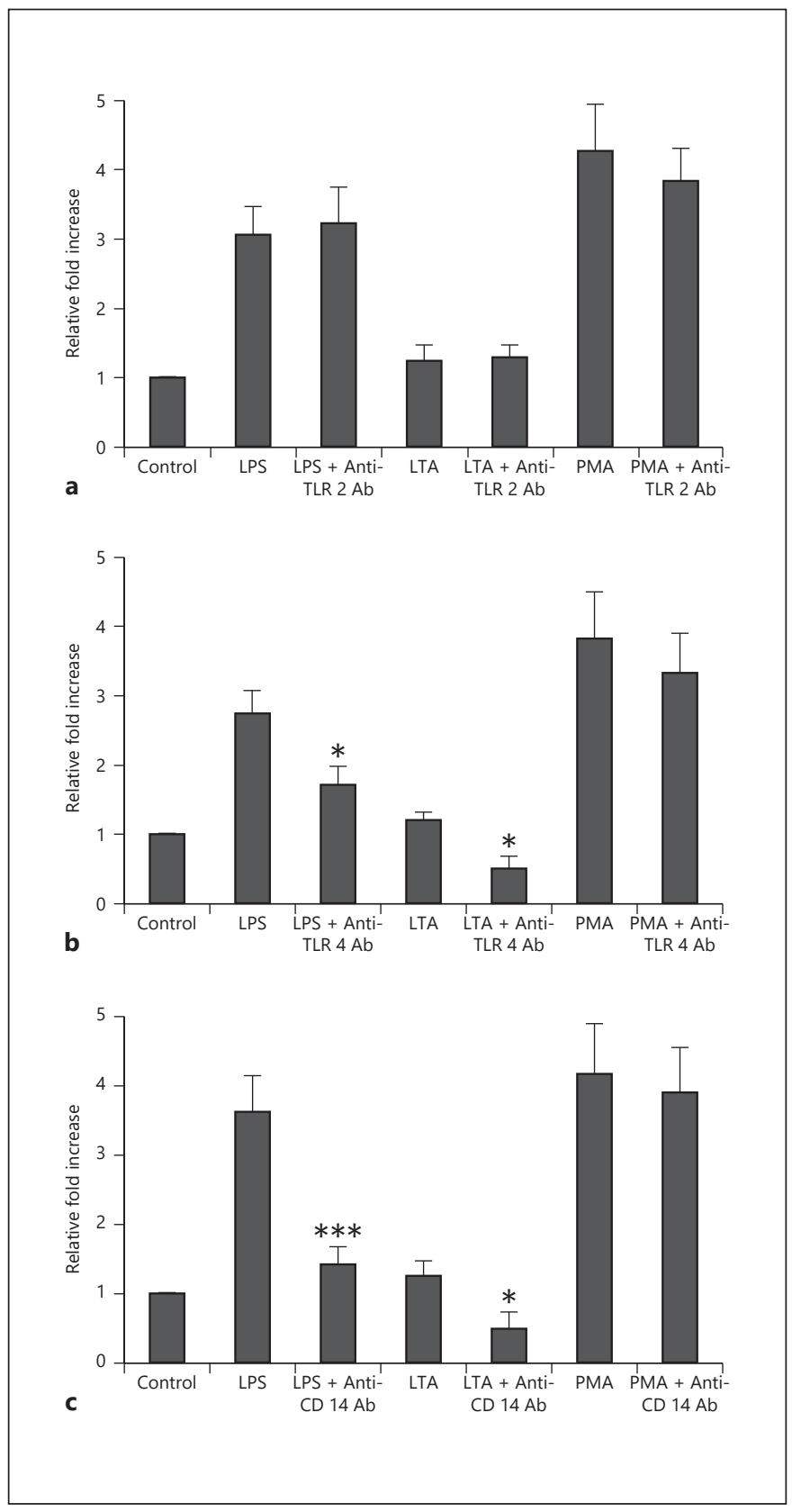

Fig. 6. Microbial components induce SZ95 sebocytes to release IL-6 by a TLR4- and CD14-dependent mechanism. LPS and LTA induction of SZ95 sebocyte IL-6 secretion was examined by ELISA. SZ95 sebocytes were pretreated with $10 \mu \mathrm{g} / \mathrm{mL}$ anti-TLR2 $\mathrm{mAb}(\mathbf{a})$, anti-TLR4 mAb (b), anti-CD14 mAb (c), or mouse IgG2a isotype control antibody for $1 \mathrm{~h}$ and then stimulated with $100 \mathrm{ng} / \mathrm{mL}$ LPS, LTA, or PMA for $24 \mathrm{~h}$. LPS-induced IL-6 release was blocked by anti-TLR4 and anti-CD14 $\mathrm{mAb}$. Anti-TLR4 and anti-CD14 mAb also slightly reduced the basal IL-6 release. Control IL-6 levels (under IgG2a isotype control antibody treatment): $506 \pm 37 \mathrm{pg} / \mathrm{mL}$. The chart shows the mean \pm SEM of 7 independent experiments. Statistical analysis was performed by Student's $t$ test using a two-tailed hypothesis. ${ }^{*} p<0.05$; ${ }^{* * *} p<0.01$. TLR, Toll-like receptor; LPS, lipopolysaccharides; LTA, lipoteichoic acid; PMA, phorbol 12-myristate 13-acetate; mAb, mouse monoclonal antibodies. 


\section{Discussion}

This study confirms previous evidence that human sebocytes express functional TLR2 and TLR4 [5, 18-20] and detects for the first time the expression of the TLR4 co-receptor CD14 in human sebocytes. We could further confirm TLR6 mRNA expression, which is known to form a heterodimeric pair with TLR2 [32]. Thus, the expression pattern of evolutionarily conserved TLR might enable human sebocytes to respond directly to microorganisms, as already shown for keratinocytes in vitro [21]. The activation of TLR and CD14 with subsequent release of cytokines, which regulate the local immune response, is a mechanism by which bacterial products may enhance inflammation in acne $[5,18-20]$.

The parallel detection of TLR2, TLR4, TLR6, and CD14 mRNA in LPS-stimulated primary human keratinocytes in our study also confirms the findings by Song et al. [17], Pivarcsi et al. [33], and Nagy et al. [21], who reported that human keratinocytes constitutively express TLR2, TLR4, and CD14 responding to low concentrations of LPS.

TLR2 and TLR4 are the most widely studied PRR. TLR2 is involved in the recognition of a variety of microbial products such as lipopeptides and lipoproteins from Gramnegative and Gram-positive bacteria as well as LTA, peptidoglycans from Gram-positive bacteria, mycobacterial lipoarabinomannan, neisserial porins [34], zymosan from yeasts [35], atypical LPS, and heat-shock proteins [36, 37]. Peptidoglycan recognition requires the TLR2/TLR6 complex, whereas recognition of LTA only needs the presence of TLR2 [16]. In contrast, TLR4 primarily mediates cellular signaling induced by Gram-negative bacteria and was characterized as a receptor specific for LPS $[17,38]$. LPS are a major component in the Gram-negative bacterial cell wall, whereas only saturated fatty acids containing LPS act as a ligand for TLR4 $[39,40]$. CD14 is a co-receptor of TLR4 but has the ability to also recognize LTA and peptidoglycans from Gram-positive bacteria [41, 42]. In our study, LPS already at low concentrations, which do not affect cell proliferation and differentiation, slightly upregulated mRNA expression of TLR2 in SZ95 sebocytes.

The marked induction of IL- $1 \alpha$, IL- $1 \beta$, IL- 6 , and IL- 8 by LPS in SZ95 sebocytes confirms earlier reports of cytokine expression in and around the pilosebaceous unit at the preclinical and initial stages of acne lesions [15, 43-45]. In addition, our experiments on blocking TLR2, TLR4, and CD14 indicate that microbial components might induce human sebocyte IL- 8 and IL- 6 release by a mechanism probably dependent on TLR4/CD14 under the conditions tested. These experiments demonstrate that microbial components are capable of directly activat-

Toll-Like Receptors in Human Sebocytes ing SZ95 sebocytes to express and release IL- 8 and IL-6 in an orchestrated TLR4- and CD14-dependent manner.

In acne, the host response to $P$. acnes may result in the production of proinflammatory cytokines and contribute to the clinical manifestations of the disease. Our data suggest that wall components of Gram-positive bacteria, such as LTA, might be less able to trigger an inflammatory cytokine response in human sebocytes than the Gram-negative bacterial product LPS, indicating the possibility that other bacteria than $P$. acnes might be responsible for the initiation of acne [46].

A major goal of acne therapy is to reduce inflammation. 13cRA is used to reduce sebaceous gland size by decreasing proliferation and inflammation $[22,47]$. Corticosteroids, such as prednisolone or dexamethasone, modify the inflammatory response in vivo and in vitro and are used in combination with retinoids in severe inflammatory acne and acne fulminans [24]. Since TLR may mediate inflammation in acne $[11,15]$, we examined whether the mechanism of anti-inflammatory activity of retinoids and corticosteroids correlates with regulation of TLR expression and function. Retinoids exhibited a rather weak overall effect on cytokine release from SZ95 sebocytes. However, IL-6, which induces lymphocyte chemotaxis and is constitutively expressed in human sebocytes, was affected by retinoids due to a significant release reduction, especially through $13 \mathrm{cRA}$ and atRA. This finding indicates a selective anti-inflammatory effect of retinoids on human sebocytes. In contrast to our findings, Liu et al. [25] reported that atRA was able to inhibit cytokine release from monocytes by downregulating TLR2 and CD14 expression and function, while TLR4-induced cytokine release was not affected. On the other hand, atRA reduced TLR2 expression in human keratinocytes while simultaneously increased the levels of IL-8 and TLR4 [48]. Evidently, the mode of retinoid-regulated gene expression depends on cell type with exertion of an anti-inflammatory effect via two pathways, one specifically affecting TLR2 and CD14 expression and another TLR-independent, the latter probably is common with human sebocytes.

As previously reported for dexamethasone [49], HC upregulated TLR2 mRNA levels, but markedly reduced TLR4 and CD14 mRNA levels. This confirms our hypothesis that TLR2 acts as a "guard" for human sebocytes, as it is upregulated after treatment with anti-inflammatory agents. HC induced an overall reduction of cytokine/ chemokine release in SZ95 sebocytes, a finding that emphasizes the global anti-inflammatory activity of corticosteroids, also on sebocytes, which is clinically obvious in severe acne $[23,24]$.

Skin Pharmacol Physiol 2021;34:19-29 DOI: $10.1159 / 000513259$ 
This study provides further support for an active role of human sebocytes in innate immune response at least through the constitutive expression of functional TLR2, TLR4, and CD14. Obviously, the expression of functional receptors for pathogen-associated molecular patterns is required not only in professional epidermal immune cells, such as Langerhans cells, but also in human sebocytes and epidermal keratinocytes to guarantee normal skin homeostasis. Since the pilosebaceous unit is in direct contact with microorganisms, it is not surprising that human sebocytes are endowed with the capacity to initiate innate immune responses. Georgel et al. [1], Nagy et al. [2], Lovászi et al. [50], and Sanford et al. [45] have provided evidence for the potential of human sebocytes to kill pathogens by various mechanisms such as the production of antibacterial lipids and the expression of antimicrobial peptides.

On the other hand, an important role for TLR2 and TLR4 receptors in the hypothalamus-pituitary adrenal axis has previously been suggested [51]. Human sebocytes express their own neuroendocrine stress system including the capacity to modify local steroid metabolism [52]. Thus, sebocytes are responsive to stress, providing a potential link to the pathogenesis of acne [53]. The present data underline the close functional interdependence of both the classical and local endocrine stress system with the key mediators of the cutaneous innate system.

In conclusion, human sebocytes express functional PRR. Microbial components use them to directly activate the cells to express a wide range of proinflammatory molecules and especially IL-8 and IL-6 in a TLR4- and CD14specific manner, thus initiating and perpetuating the activation of both innate and adaptive immune responses in human skin [3]. Retinoids, but mostly corticosteroids, also use this pathway to exhibit anti-inflammatory effects.

\section{Acknowledgements}

Holger Seltmann has significantly contributed to the research presented in this manuscript. We acknowledge Nahid H. Hakiy, Birgit Bogdanoff, Aristotelis Nastos, and Rhodri Walters for skillful technical assistance and experimental support, respectively.

\section{Statement of Ethics}

The authors have no ethical conflicts to disclose. The reported research was conducted ethically in accordance with the World Medical Association Declaration of Helsinki.

\section{Conflict of Interest Statement}

The authors declare no conflicts of interest regarding this manuscript. C.C. Zouboulis is the owner of an international patent on the immortalized human sebaceous gland cell line SZ95 (WO0046353).

\section{Funding Sources}

This work was supported in part by research grants of the Bundesinstitut für gesundheitlichen Verbraucherschutz und Veterinärmedizin (BgVV Z 5.1-1328-156) and the Sonnenfeld Stiftung to Christos C. Zouboulis and the Deutsche Forschungsgemeinschaft (FOR 441/1-1) to Stefan R. Bornstein.

\section{Author Contributions}

Study design: C.C. Zouboulis. Performance of the experiments: M.K. Oeff, H. Naoki, and E. Makrantonaki. Statistical analysis: M.K. Oeff. Preparation and revision of the manuscript: C.C. Zouboulis, M.K. Oeff, H. Naoki, E. Makrantonaki, and S.R. Bornstein.

\section{References}

1 Georgel P, Crozat K, Lauth X, Makrantonaki E, Seltmann H, Sovath S, et al. A Toll-like receptor 2-responsive lipid effector pathway protects mammals against skin infections with Grampositive bacteria. Infect Immun. 2005;73(8): 4512-21.

2 Nagy I, Pivarcsi A, Kis K, Koreck A, Bodai L, McDowell A, et al. Propionibacterium acnes and lipopolysaccharide induce the expression of antimicrobial peptides and proinflammatory cytokines/chemokines in human sebocytes. Microbes Infect. 2006;8(8):2195-205.

3 Mattii M, Lovászi M, Garzorz N, Atenhan A, Quaranta M, Lauffer F, et al. Sebocytes contribute to skin inflammation by promoting the differentiation of T helper 17 cells. Br J Dermatol. 2018;178(3):722-30.
4 Janeway CA Jr, Medzhitov R. Innate immune recognition. Annu Rev Immunol. 2002;20:197216.

5 Töröcsik D, Kovács D, Póliska S, SzentkeresztyKovács Z, Lovászi M, Hegyi K, et al. Genome wide analysis of TLR2- and TLR4-activated SZ95 sebocytes reveals a complex immune-competence and identifies serum amyloid $\mathrm{A}$ as a marker for activated sebaceous glands. PLoS One. 2018;13(6): 0198323.

6 Zouboulis CC, Beutler C, Merk HF, Baron JM. RIS-1/psoriasin expression in epithelial skin cells indicates their selective role in innate immunity and in inflammatory skin diseases including acne. Dermatoendocrinol. 2017;9(1):e1338993.

7 Rock FL, Hardiman G, Timans JC, Kastelein RA, Bazan JF. A family of human receptors structur- ally related to Drosophila Toll. Proc Natl Acad Sci U S A. 1998;95(2):588-93.

8 Kawai T, Akira S. TLR signaling. Cell Death Differ. 2006;13(5):816-25.

9 Jugeau S, Tenaud I, Knol AC, Jarrousse V, Quereux G, Khammari A, et al. Induction of toll-like receptors by Propionibacterium acnes. Br J Dermatol. 2005;153(6):1105-13.

10 Kim J. Review of the innate immune response in acne vulgaris: activation of Toll-like receptor 2 in acne triggers inflammatory cytokine responses. Dermatology. 2005;211(3):193-8.

11 Kim J, Ochoa MT, Krutzik SR, Takeuchi O, Uematsu S, Legaspi AJ, et al. Activation of toll-like receptor 2 in acne triggers inflammatory cytokine responses. J Immunol. 2002;169(3):153541.

Zouboulis/Oeff/Hiroi/Makrantonaki/ Bornstein
Skin Pharmacol Physiol 2021;34:19-29

DOI: $10.1159 / 000513259$ 
12 Jeremy AH, Holland DB, Roberts SG, Thomson $\mathrm{KF}$, Cunliffe WJ. Inflammatory events are involved in acne lesion initiation. J Invest Dermatol. 2003;121(1):20-7.

13 Makrantonaki E, Ganceviciene R, Zouboulis C. An update on the role of the sebaceous gland in the pathogenesis of acne. Dermatoendocrinol. 2011;3(1):41-9.

14 Zouboulis CC, Eady A, Philpott M, Goldsmith LA, Orfanos C, Cunliffe WC, et al. What is the pathogenesis of acne? Exp Dermatol. 2005; 14(2): $143-52$.

15 Alestas T, Ganceviciene R, Fimmel S, MüllerDecker K, Zouboulis CC. Enzymes involved in the biosynthesis of leukotriene B4 and prostaglandin E2 are active in sebaceous glands. J Mol Med. 2006;84(1):75-87.

16 Kollisch G, Kalali BN, Voelcker V, Wallich R, Behrendt H, Ring J, et al. Various members of the Toll-like receptor family contribute to the innate immune response of human epidermal keratinocytes. Immunology. 2005;114(4):531-41.

17 Song PI, Park YM, Abraham T, Harten B, Zivony A, Neparidze N, et al. Human keratinocytes express functional CD14 and Toll-like receptor 4. J Invest Dermatol. 2002;119(2):424-32.

18 Lee SE, Kim JM, Jeong MK, Zouboulis CC, Lee $\mathrm{SH}$. 11 $\beta$-hydroxysteroid dehydrogenase type 1 is expressed in human sebaceous glands and regulates glucocorticoid-induced lipid synthesis and Toll-like receptor 2 expression in SZ95 sebocytes. Br J Dermatol. 2013;168(1):47-55.

19 Mirdamadi Y, Thielitz A, Wiede A, Goihl A, Papakonstantinou E, Hartig R, et al. Insulin and insulin-like growth factor-1 can modulate the phosphoinositide-3-kinase/Akt/FoxO1 pathway in SZ95 sebocytes in vitro. Mol Cell Endocrinol. 2015;415:32-44.

20 Selway JL, Kurczab T, Kealey T, Langlands K. Toll-like receptor 2 activation and comedogenesis: implications for the pathogenesis of acne. BMC Dermatol. 2013;13:10.

21 Nagy I, Pivarcsi A, Koreck A, Széll M, Urbán E, Kemény L. Distinct strains of Propionibacterium acnes induce selective human beta-defensin- 2 and interleukin- 8 expression in human keratinocytes through Toll-like receptors. J Invest Dermatol. 2005;124(5):931-8.

22 Zouboulis CC. Exploration of retinoid activity and the role of inflammation in acne: issues affecting future directions for acne therapy. J Eur Acad Dermatol Venereol. 2001;15(Suppl 3):637.

23 Zouboulis CC, Bettoli V. Management of severe acne. Br J Dermatol. 2015;172(Suppl 1):27-36.

24 Massa AF, Burmeister L, Bass D, Zouboulis CC. Acne fulminans: treatment experience from 26 patients. Dermatology. 2017;233(2-3):136-40.

25 Liu PT, Krutzik SR, Kim J, Modlin RL. Cutting edge: all-trans retinoic acid down-regulates TLR2 expression and function. J Immunol. 2005; 174(5):2467-70.

26 Dispenza MC, Wolpert EB, Gilliland KL, Dai JP, Cong Z, Nelson AM, et al. Systemic isotretinoin therapy normalizes exaggerated TLR-2-mediat- ed innate immune responses in acne patients. J Invest Dermatol. 2012;132(9):2198-205.

27 RRID:CVCL_9803. Available from: https://web. expasy.org/cellosaurus/CVCL_9803.

28 Zouboulis CC, Seltmann H, Neitzel H, Orfanos CE. Establishment and characterization of an immortalized human sebaceous gland cell line (SZ95). J Invest Dermatol. 1999;113(6):1011-20.

29 CLS Cat\# 300209/p671_HL-60, RRID: CVCL_0002. Available from: https://web.expasy.org/cellosaurus/CVCL_0002.

30 Asemissen AM, Nagorsen D, Keilholz U, Letsch A, Schmittel A, Thiel E, et al. Flow cytometric determination of intracellular or secreted IFNgamma for the quantification of antigen reactive T cells. J Immunol Methods. 2001;251(1-2): 101-8.

31 Hiroi N, Wong ML, Licinio J, Park C, Young M, Gold PW, et al. Expression of corticotropin releasing hormone receptors type I and type II mRNA in suicide victims and controls. Mol Psychiatry. 2001;6(5):540-6.

32 Ozinsky A, Underhill DM, Fontenot JD, Hajjar $\mathrm{AM}$, Smith KD, Wilson CB, et al. The repertoire for pattern recognition of pathogens by the innate immune system is defined by cooperation between Toll-like receptors. Proc Natl Acad Sci U S A. 2000;97(25):13766-71.

33 Pivarcsi A, Bodai L, Réthi B, KenderessySzabó A, Koreck A, Széll M, et al. Expression and function of Toll-like receptors 2 and 4 in human keratinocytes. Int Immunol. 2003; 15(6):721-30.

34 Massari P, Henneke P, Ho Y, Latz E, Golenbock DT, Wetzler LM. Cutting edge: immune stimulation by neisserial porins is Toll-like receptor 2 and MyD88 dependent. J Immunol. 2002; 168(4):1533-7.

35 Aderem A, Ulevitch RJ. Toll-like receptors in the induction of the innate immune response. $\mathrm{Na}$ ture. 2000;406(6797):782-7.

36 Brightbill HD, Libraty DH, Krutzik SR, Yang RB, Belisle JT, Bleharski JR, et al. Host defense mechanisms triggered by microbial lipoproteins through Toll-like receptors. Science. 1999; 285(5428):732-6.

37 Yoshimura A, Lien E, Ingalls RR, Tuomanen E, Dziarski R, Golenbock D. Cutting edge: recognition of Gram-positive bacterial cell wall components by the innate immune system occurs via Toll-like receptor 2. J Immunol. 1999;163(1):15.

38 Hoshino K, Takeuchi O, Kawai T, Sanjo H, Ogawa T, Takeda Y, et al. Cutting edge: Toll-like receptor 4 (TLR4)-deficient mice are hyporesponsive to lipopolysaccharide: evidence for TLR4 as the LPS gene product. J Immunol. 1999;162(7): 3749-52.

39 Lee JY, Sohn KH, Rhee SH, Hwang D. Saturated fatty acids, but not unsaturated fatty acids, induce the expression of cyclooxygenase- 2 mediated through Toll-like receptor 4. J Biol Chem. 2001;276(20):16683-9.

40 Lovászi M, Mattii M, Eyerich K, Gácsi A, Csányi E, Kovács D, et al. Sebum lipids influence mac- rophage polarization and activation. Br J Dermatol. 2017;177(6):1671-82.

41 Manukyan M, Triantafilou K, Triantafilou M, Mackie A, Nilsen N, Espevik T, et al. Binding of lipopeptide to $\mathrm{CD} 14$ induces physical proximity of CD14, TLR2 and TLR1. Eur J Immunol. 2005; 35(3):911-21.

42 Vowels BR, Yang S, Leyden JJ. Induction of proinflammatory cytokines by a soluble factor of Propionibacterium acnes: implications for chronic inflammatory acne. Infect Immun. 1995;63(8):3158-65.

43 Guy R, Kealey T. Modelling the infundibulum in acne. Dermatology. 1998;196(1):32-7.

44 Huang YC, Yang CH, Li TT, Zouboulis CC, Hsu HC. Cell-free extracts of Propionibacterium acnes stimulate cytokine production through activation of p38 MAPK and Toll-like receptor in SZ95 sebocytes. Life Sci. 2015;139: 123-31.

45 Sanford JA, O’Neill AM, Zouboulis CC, Gallo RL. Short-chain fatty acids from cutibacterium acnes activate both a canonical and epigenetic inflammatory response in human sebocytes. J Immunol. 2019;202(6):1767-76.

46 Xu DT, Yan JN, Liu W, Hou XX, Zheng Y, Jiang $\mathrm{WW}$, et al. Is human sebum the source of skin follicular ultraviolet-induced red fluorescence? A cellular to histological study. Dermatology. 2018;234(1-2):43-50.

47 Tsukada M, Schröder M, Roos TC, Chandraratna RA, Reichert U, Merk HF, et al. 13-cis Retinoic acid exerts its specific activity on human sebocytes through selective intracellular isomerization to all-trans retinoic acid and binding to retinoid acid receptors. J Invest Dermatol. 2000; 115(2):321-7.

48 Erdei L, Bolla BS, Bozó R, Tax G, Urbán E, Kemény L, et al. TNIP1 regulates cutibacterium acnes-induced innate immune functions in epidermal keratinocytes. Front Immunol. 2018;9: 2155.

49 Homma T, Kato A, Hashimoto N, Batchelor J, Yoshikawa M, Imai S, et al. Corticosteroid and cytokines synergistically enhance toll-like receptor 2 expression in respiratory epithelial cells. Am J Respir Cell Mol Biol. 2004;31(4):4639.

50 Lovászi M, Szegedi A, Zouboulis CC, Törőcsik D. Sebaceous-immunobiology is orchestrated by sebum lipids. Dermatoendocrinol. 2017;9(1): e1375636.

51 Bornstein SR, Schumann RR, Rettori V, McCann SM, Zacharowski K. Toll-like receptor 2 and Toll-like receptor 4 expression in human adrenals. Horm Metab Res. 2004;36(7):470-3.

52 Zouboulis CC, Seltmann H, Hiroi N, Chen W, Young M, Oeff M, et al. Corticotropin-releasing hormone: an autocrine hormone that promotes lipogenesis in human sebocytes. Proc Natl Acad Sci U S A. 2002;99(10):7148-53.

53 Ganceviciene R, Graziene V, Fimmel S, Zouboulis CC. Involvement of the corticotropin-releasing hormone system in the pathogenesis of acne vulgaris. Br J Dermatol. 2009;160(2):345-52. 